\title{
AaMYB3 interacts with AabHLH1 to regulate proanthocyanidin accumulation in Anthurium andraeanum (Hort.) - another strategy to modulate pigmentation
}

\author{
Chonghui $\mathrm{Li}^{1,2}$, Jian Qiü ${ }^{3}$ Surong Huang ${ }^{1,2}$, Junmei Yin ${ }^{1,2}$ and Guangsui Yang ${ }^{1,2}$
}

\begin{abstract}
Proanthocyanidins (PAs), also known as "condensed tannins", are colorless metabolites produced through the flavonoid pathway that are involved in stress resistance in plants. Because PAs are involved in the anthocyanin biosynthetic pathway, they play a role in the modification of pigmentation conferred by anthocyanins in ornamental organs. In this study, we isolated the gene and functionally characterized an R2R3-MYB transcription factor (TF), AaMYB3, and a basic helix-loop-helix TF, AabHLH1, from Anthurium andraeanum (Hort.), a typical tropical flower. $A a M Y B 3$ is primarily expressed in the spathe and negatively correlates with anthocyanin accumulation. A complementation test in an Arabidopsis tt8 mutant showed that AabHLH1 successfully restores the PA-deficient seed coat phenotype. The ectopic overexpression of AaMYB3 alone or its coexpression with $A a b H L H 1$ in transgenic tobacco resulted in light pink or even pale-pink corolla limbs by reducing their anthocyanin levels and greatly enhancing their accumulation of PAs. This overexpression of the anthurium TF genes upregulated the late anthocyanin enzymeencoding genes (NtDFR and NtANS) and the key PA genes (NtLAR and NtANR) in transgenic tobacco. The interaction between AaMYB3 and the AabHLH1 protein was confirmed using yeast two-hybrid $(Y 2 H)$ and bimolecular fluorescence complementation (BiFC) assays. In the developing red spathes of the cultivars "Vitara" and "Tropical", the expression of AaMYB3 was closely linked to PA accumulation, and AaMYB3 was coexpressed with AaCHS, AaF3H, AaDFR, AaANS, AaLAR, and AaANR. The expression pattern of AabHLH1 was similar to that of AaF3'H. Our results suggest that AaMYB3 and AabHLH1 are involved in the regulation of PA biosynthesis in anthurium and could potentially be used to metabolically engineer PA biosynthesis in plants.
\end{abstract}

\section{Introduction}

Proanthocyanidins (PAs), the end products of the flavonoid biosynthetic pathway, occur in the fruits, bark,

Correspondence: Junmei Yin (yinjunmei2011@sina.com) or Guangsui Yang (13976572870@163.com)

${ }^{1}$ Tropical Crops Genetic Resources Institute, the Chinese Academy of Tropical Agricultural Sciences (CATAS) / Key Laboratory of Crop Gene Resources and Germplasm Enhancement in Southern China, Ministry of Agriculture, Danzhou 571737, China

${ }^{2}$ The Engineering Technology Research Center of Tropical Ornamental Plant Germplasm Innovation and Utilization, Hainan Province, Danzhou 571737,

China

Full list of author information is available at the end of the article.

These authors contributed equally: Chonghui Li, Jian Qiu leaves, and seeds of many plants ${ }^{1}$. PAs have strong antioxidant properties, and their primary function in plants is to defend against pathogens, insects, diseases, and larger herbivores ${ }^{1,2}$. PAs, also called "condensed tannins", confer astringency upon plants that originally served as forage and function as herbivore feeding deterrents ${ }^{1,3}$. The dietary PAs in wine, fruit juices, teas, and cocoa contribute to their taste and health benefits through their antioxidant and radical-scavenging functions and their anti-inflammatory activities ${ }^{4}$. Therefore, there is strong interest in the molecular biosynthesis and metabolic engineering of PAs in crops and fruits ${ }^{5,6}$.

\section{(c) The Author(s) 2019}

(c) (i) Open Access This article is licensed under a Creative Commons Attribution 4.0 International License, which permits use, sharing, adaptation, distribution and reproduction in any medium or format, as long as you give appropriate credit to the original author(s) and the source, provide a link to the Creative Commons license, and indicate if changes were made. The images or other third party material in this article are included in the article's Creative Commons license, unless indicated otherwise in a credit line to the material. If material is not included in the article's Creative Commons license and your intended use is not permitted by statutory regulation or exceeds the permitted use, you will need to obtain permission directly from the copyright holder. To view a copy of this license, visit http://creativecommons.org/licenses/by/4.0/. 
PA biosynthesis shares the same upstream pathway with anthocyanins, although they are subsequently synthesized as the oligomeric or polymeric end products of one of several branches of the flavonoid pathway in the final catalytic steps ${ }^{4}$. The formation of the flavan-3-ols begins with the dihydroflavanol 4-reductase (DFR)mediated reduction of dihydroflavonols to leucoanthocyanidins, which are then reduced by leucoanthocyanidin reductase (LAR) to catechin. The other way that flavan-3ols (epicatechin) are formed by the reduction of anthocyanidin, which is converted from leucoanthocyanidins by anthocyanidin synthase (ANS), which is catalyzed by anthocyanidin reductase (ANR). Flavonoid biosynthesis is primarily controlled by transcription factors (TFs) that regulate the expression of the genes encoding the biosynthetic enzymes in the associated pathways. In most plants, the process is conservatively regulated by the MBW protein complex formed by the combination of R2R3-MYB, basic helix-loop-helix protein (bHLH) and the WD40 repeat-containing protein (WDR) ${ }^{7}$. The regulation of PA biosynthesis by the MBW complex has been well characterized in Arabidopsis with the TT2 (MYB)-TT8 (bHLH)-TTG1 (WDR) model ${ }^{8}$. The ternary transcription protein activates the late anthocyanin and PA-specific genes, including DFR, ANS, and $B A N$ (also known as $A N R)^{8-10}$. The homologs of TT2, TT8, and TTG1 in other plants have since been discovered. For example, in strawberry, FaMYB9/FaMYB11, FabHLH3, and FaTTG1 form a complex that upregulates the expression of $A N S$ and $L A R$, therefore increasing the levels of $\mathrm{PAs}^{11}$. In persimmon, DkMYB2 and DkMYB4 interact with both DkMYC1 (bHLH) and DkWDR1 to regulate PA accumulation in the fruit ${ }^{12}$. The WDR proteins in the MBW complex are thought to confer a docking platform for the MYB-bHLH interaction. Many studies have suggested that R2R3MYB is a key factor that determines the activity that induces or represses the transcription of the PA biosynthetic genes. For example, in grapevine, VvMYBPA $1^{13}$, VvMYBPA $^{14}$, and VvMYBPAR ${ }^{15}$ promote PA accumulation, and $\mathrm{VvMYBC} 2-\mathrm{L}^{16}$ negatively regulates $\mathrm{PA}$ accumulation by downregulating the expression of the PA genes. Therefore, much attention has been paid to the role of the MYB TF in the PA transcriptional regulation process. Many MYBs have been identified as PA regulators, such as poplar PtMYB134 ${ }^{17}$, Trifolium arvense TaMYB14 ${ }^{18}$, apple MdMYB9 ${ }^{19}$, peach PpMYB7 $^{20}$, and even in the ornamental plants coleus $\left(\mathrm{SsMYB3}^{21}\right)$ and Malus crabapple (MdMYB12 $\mathrm{b}^{22}$ ). Many of these genes are considered suitable candidates to metabolically engineer PA biosynthesis in plants, because MYBs regulate multiple key enzyme-encoding genes, and therefore have advantages over single key enzymes in such genebased strategies ${ }^{21}$.
Anthurium andraeanum (Hort.) is a well-known tropical flower with a colorful spathe and spadix and is produced commercially as a cut flower or potted plant ${ }^{23}$. Anthurium has a long horticultural history and is very important in worldwide trade. The color of the spathe and spadix is one of the most important traits of anthuriums. The anthocyanins cyanidin, pelargonidin and peonidin, in the form of anthocyanidin 3-rutinoside, are primarily responsible for the red, purple, pink, orange, and coral coloration of the anthuriums ${ }^{24}$. The biosynthetic pathways and the expression pattern of the key enzyme genes in anthocyanin biosynthesis have been characterized in anthurium ${ }^{23,25,26}$. However, in the MBW complex, only the MYB genes have so far been identified in anthurium by their ectopic expression. AaMYB1 ${ }^{27}$ is involved in the positive regulation of anthocyanin biosynthesis, and AnAN2 may act as a negative regulator of anthocyanin production ${ }^{28}$. We previously isolated the R2R3-MYB gene AaMYB2 and demonstrated that $A a M Y B 2$ expression is closely related to anthocyanin accumulation and that AaMYB2 primarily contributes to the regulation of anthocyanin biosynthesis in the anthurium spathes and leaves ${ }^{29}$. However, the regulatory mechanism of the biosynthesis of flavonoids, including anthocyanins and PAs, in anthurium is still unclear.

PAs are colorless flavonoid polymers that are later pathway products downstream from anthocyanins. The ectopic expression of ANR in Nicotiana tabacum (tobacco) flowers and Arabidopsis leaves resulted in the loss of anthocyanins and the accumulation of PAs, suggesting that the excessive accumulation of PAs causes a reduction in anthocyanin ${ }^{30,31}$. Similarly, the expression pattern of $A N R$ correlates negatively with anthocyanin accumulation in the anthurium spathe ${ }^{32}$. Therefore, PA accumulation in these ornamental plant organs dilutes the pigmentation contributed by anthocyanins, thus playing an important role in coloration. However, very few studies have reported the key structural and regulatory genes involved in the PA biosynthetic pathway in ornamental plants. Therefore, the isolation and characterization of the genes that regulate this pathway are necessary to understand the molecular regulation of PA and anthocyanin biosynthesis in this species ${ }^{21}$.

In this study, we present the isolation of the first gene encoding a bHLH protein and one gene encoding an R2R3-MYB TF in anthurium, designated AabHLH1 and AaMYB3, respectively. A phylogenetic analysis indicated that AaMYB3 and AabHLH1 are homologous to the Arabidopsis PA regulators AtTT2 and AtTT8, respectively. The functions of AaMYB3 and AabHLH1 were demonstrated with a complementation test in an Arabidopsis mutant and their exogenous expression in tobacco. Our results demonstrate that AaMYB3 interacts with AabHLH1 and that they are involved in PA biosynthesis 
in anthurium. Based on these results and our previous study, we propose a model of the regulation of anthocyanin and PA accumulation in the anthurium spathe, which extends our understanding of the whole flavonoid metabolic pathway in anthurium. These two TFs, AaMYB3 and AabHLH1, may also be useful in the metabolic engineering of PA biosynthesis in plants.

\section{Materials and methods}

\section{Plant materials}

Mature 6-year-old plants of seven A. andraeanum (Hort.) cultivars maintained in a shade greenhouse at the Tropical Flower Resource Garden, Tropical Crops Genetic Resources Institute, Chinese Academy of Tropical Agricultural Sciences (Danzhou, Hainan province, China) were used in this study. The cultivars were "Tropical" and "Vitara" (red-spathed), "Pink Champion" (pink), "Cheers" (light pink), "Rapido" (purple), "Acropolis" (white), and "Midori" (green). Floral tissue samples, including the spathe and the spadix, were collected between 9 A.M. and 10 A.M. in November 2016 from the developmental stages 1 to 5 of the spathe as described by $\mathrm{Li}$ et $\mathrm{al}^{29}$. They were stage 1, the flower (including the spathe and spadix) fully protruded from the protection sheath; stage 2, the floral peduncle elongated but the spathe tightly furled; stage 3, the spathe was half unfurled; stage 4, the spathe was newly fully expanded, and stage 5, the color at the lower two-thirds of spadix became shallow. Simultaneously, the newly fully expanded brown leaves and green mature leaves and the peduncle of flower at stage 4 of "Tropical" were also obtained. Three biological replicates (three distinct spathes or spadix or leaves) within five random plants were selected and used for the subsequent metabolite and RNA analyses.

Nicotiana tabacum cv. Wisconsin 38 was used in the gene overexpression experiments. All the tobacco plants were grown in a $50 \%$ shade greenhouse under natural sunlight. The corolla limbs of the $\mathrm{T}_{1}$ transgenic tobacco plants were collected between 9 A.M. and $10 \mathrm{~A}$. M. in April 2017. Three biological replicates (three distinct tobacco flowers) within five plants were selected and used for the subsequent metabolite and RNA analyses.

Arabidopsis thaliana ecotype "Columbia" was used as the wild type control. In addition, the $t t 2$ and $t t 8$ Arabidopsis mutants (SALK 005260 and SALK 063334, respectively) obtained from the Arabidopsis Biological Resource Center, were used as the background for the genetic transformation and the negative control. All the Arabidopsis plants were grown in 14-h days and 10-h nights at $23^{\circ} \mathrm{C}$ in a growth chamber.

\section{Anthocyanin, flavonoid, and polyphenol contents measurement}

The extraction and the content measurement of the anthocyanins were performed as described by Li et al. ${ }^{29}$. The total flavonoids and polyphenols were extracted and measured as described previously ${ }^{33}$ with slight modifications. The extracts were prepared using fresh tissue samples extracted with methanol. The experiment was repeated three times for each sample.

\section{PA extraction and determination}

The dimethylaminocinnamaldehyde (DMACA) stain method was used to evaluate the PA content. Soluble PAs from fresh anthurium tissues and tobacco corolla limbs were extracted and measured as described previously ${ }^{34}$. Epicatechin was used as a standard for PA quantification $^{35}$. The experiment was repeated three times for each sample.

\section{RNA isolation and CDNA synthesis}

The total RNA was extracted from anthurium tissues using an RNAprep Pure Plant Kit (Polysaccharides \& Polyphenolics-rich) (TIANGEN, Beijing, China). The total RNA from the Arabidopsis leaves and tobacco corolla limbs was extracted using a Plant Total RNA Isolation Kit (FOREGENE, Chengdu, China). The cDNA was synthesized according to the manufacturer's instructions for the RevertAid First Strand cDNA Synthesis Kit (Thermo Scientific, Waltham, MA, USA).

\section{Isolation of the full-length CDNA of AaMYB3 and AabHLH1 and their sequence analysis}

One MYB unigene and one bHLH unigene were selected from the mixed floral and foliar transcriptome database of anthurium ${ }^{29}$, which were annotated as AtTT2-like and AtTT8-like transcription factors, respectively. The unigenes were designated as AaMYB3 (GenBank accession no. MH349476) and AabHLH1 (accession no. MH349477). The full-length cDNA of $A a M Y B 3$ and AabHLH1 was isolated from the "Tropical" spathe with reverse transcription (RT)-PCR using primers designed according to the transcriptome data: forward $5^{\prime}$-AT GGGCAGGAGACCCTGTT-3', reverse 5'-CGCCATTA CTTCACCCATTC-3' for AaMYB3, and forward 5'-AGG AGGGGTAGTTGAGCAGGT- ${ }^{\prime}$, reverse $5^{\prime}$-TCATGCT CTAAGCATGTCACGA-3' for AabHLH1. PCR-amplified products were cloned into the T/A cloning vector $\mathrm{PMD} 18$ $\mathrm{T}$ (TaKaRa, Dalian, China) and sequenced. The full length of the amino acid sequences deduced for AaMYB3 and AabHLH1 were constructed using the ClustalX2 and the MEGA 5.05 programs (the Neighbor-Joining method with 1000 bootstrap replications) for sequence alignment and phylogenetic analysis, respectively. 


\section{Quantitative real-time PCR (q-PCR) analysis}

The q-PCR analysis was conducted as previously described $^{29}$. The relative expression of the anthurium genes was normalized to the expression of the cyclophilin gene $(A a C Y P)$ and ubiquitin family protein gene (AaUBQ5) as described by Gopaulchan et al. $^{36}$. The AaANR (GenBank accession no. MH349478) and AaLAR (MH349479) nucleotide sequences were obtained from our transcriptome database ${ }^{29}$, which shared extremely high identity in the open reading frame (ORF) $(99.4 \%$ and 99.2\%) with the related species Anthurium amnicola ANR (GDJX01012972.1) and LAR (GDJX01014607.1), respectively.

The relative expression of the tobacco genes was normalized to the expression of the actin gene (NbACT) and the ribosomal protein L25 gene (NtL25) as described by Pérez-Díaz et $\mathrm{al}^{37}$. The relative expression of the Arabidopsis genes was normalized to the expression of AtActin and AtUBQ1 as described by Matsui et al. ${ }^{38}$. and Han et al. ${ }^{39}$. The sequences of all the primers used in q-PCR were listed in Table S1. Triplicates of each reaction were performed.

\section{Overexpression vector construction and plant transformation}

The fragments of AaMYB3 and AabHLH1 that contain the ORF were separately cloned into the plant binary vector pCXSN (T-Vector) for constitutive gene expression as described by Chen et al. ${ }^{40}$. The resulting vector pCXSN-AaMYB3 and pCXSN-AabHLH1 were transferred into Agrobacterium tumefaciens strain EHA105 using the freeze-thaw method, which was used in plant transformation. The genetic transformation of the Arabidopsis plants was based on the floral dip method ${ }^{41}$. The Agrobacterium strain EHA105 harboring the recombinant plasmids pCXSN-AaMYB3 was used to transform the Arabidopsis tt2 mutant plants, and AabHLH1 was transformed into the Arabidopsis $t t 8$ mutant plants. Seeds harvested from the transformed plants $\left(\mathrm{T}_{0}\right)$ were germinated on $1 / 2$ MS media containing $35 \mathrm{mg} / \mathrm{L}$ hygromycin. Positive homozygous progenies were selected and confirmed using genomic PCR. The seeds of the $\mathrm{T}_{3}$ progeny Arabidopsis plants were observed.

The genetic transformation of tobacco with AaMYB3 and AabHLH1 was accomplished using Agrobacteriummediated transformation ${ }^{42}$. The transgenic plants were selected by hygromycin resistance and once rooted were transferred to soil and grown in the greenhouse. Positive transgenic $T_{1}$ progeny tobacco plants identified by genomic PCR and q-PCR were used for further studies. Transgenic tobacco plants ( $T_{0}$ progeny, three lines of each gene transformation) separately expressing AaMYB3 and AabHLH1 were crossed in both the $q \times 0^{\star}$ and $0 \times q$ directions. The $F_{1}$ seeds of the AaMYB3 $\times$ AabHLH1 plants were harvested and germinated. The seedlings were selected using genomic PCR and q-PCR, and a total of 49 plants expressing both AaMYB3 and AabHLH1 were obtained for further studies.

\section{Yeast two-hybrid (Y2H) assay}

The $\mathrm{Y} 2 \mathrm{H}$ assay to investigate the interactions between AaMYBs and AabHLH1 was performed as described by Nakatsuka et al. ${ }^{43}$. The full-length CDS of AaMYB3 and $A a M Y B 2$ or $A a b H L H 1$ were cloned into the pGADT7 vector or pGBKT7 vector (Clontech, Mountain View, US), respectively. The yeast transformation and clones selection were performed as previously described ${ }^{44}$. The interaction of AtMYB75 and AtTT8 from Arabidopsis was employed as a positive control ${ }^{45}$.

\section{Bimolecular fluorescence complementation (BiFC) assay}

The full-length CDS of $A a M Y B 3$ and $A a M Y B 2$ or $A a b H L H 1$ were cloned into the binary yellow fluorescent protein (YFP) BiFC vectors pXY106 (nYFP) or pXY104 $(\mathrm{cYFP})^{46}$, respectively, resulting in the recombinant plasmids AaMYB3-nYFP, AaMYB2-nYFP, and AabHLH1cYFP. The empty vectors and the recombinant plasmids were transferred into A. tumefaciens GV3101 using the freeze-thaw method and were transiently expressed in Nicotiana benthamiana leaf by agroinfiltration. Two days after transformation, the YFP signals were examined in the transfected cells using a Zeiss780 confocal microscope (Zeiss, Jena, Germany). The gene AT1G16610 (encoding SR45) from Arabidopsis was coexpressed as a fusion to the red fluorescent protein (RFP) with the target genes in $N$. benthamiana leaf cells and was used as a nuclear localization marker ${ }^{47}$. The interaction of AtMYB75 and AtTT8 from Arabidopsis was employed as a positive control $^{45}$.

\section{DMACA Staining}

Dry Arabidopsis seeds were stained with the dimethylaminocinnamaldehyde (DMACA, Sigma) reagent (2\% [w/v] DMACA in $3 \mathrm{M} \mathrm{HCl} / 50 \%$ [w/v] methanol) for 2 days and washed several times with $70 \%$ ethanol $(\mathrm{v} / \mathrm{v})$ as described by $\mathrm{Abrahams}^{48}$. The stained seeds were photographed using a Leica M205FA microscope equipped with a DFc450c camera (Leica Microsystems, Solms, Germany).

\section{Results}

Sequences of $A a M Y B 3$ and $A a b H L H 1$ and a phylogenetic analysis

Several unigenes associated with the transcriptional control of the flavonoids and PA biosynthesis were selected from our transcriptome data according to annotations determined with BLASTx searches in the National Center for Biotechnology Information (NCBI) 
a

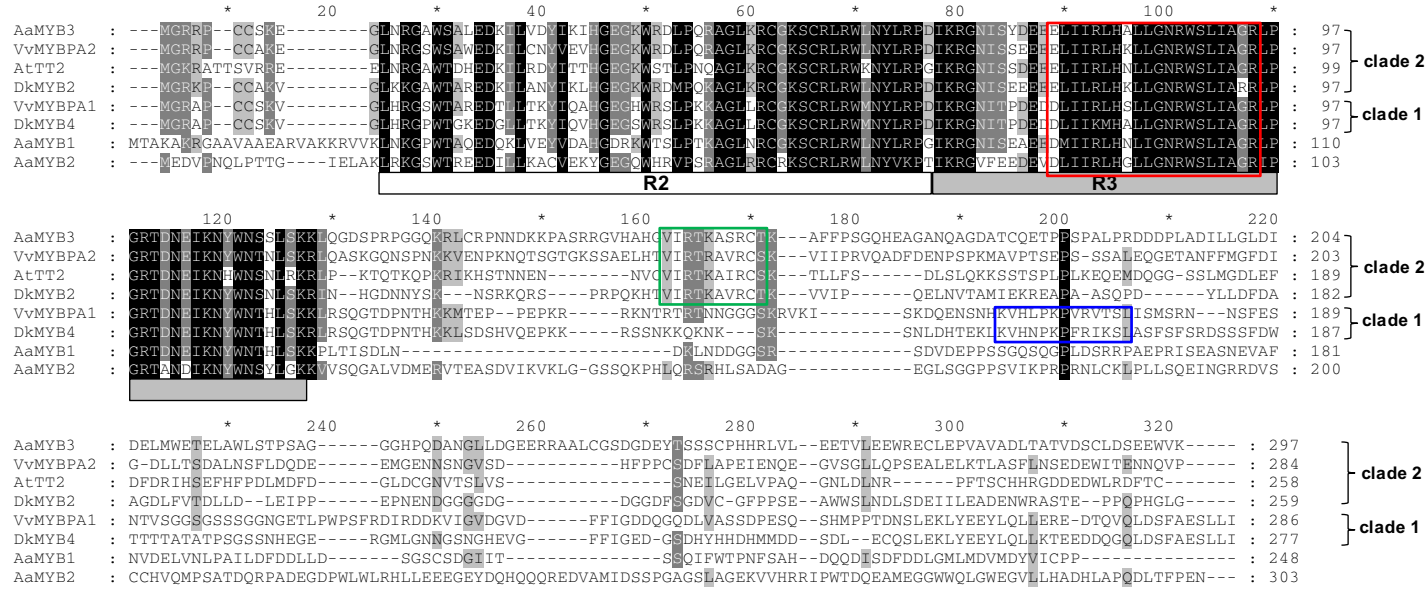

b

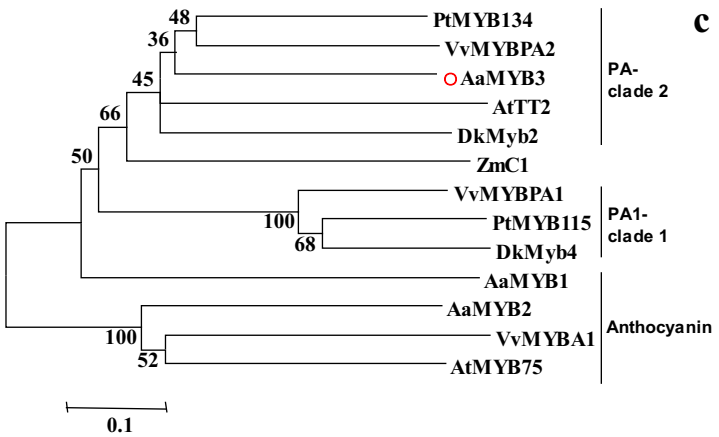

c

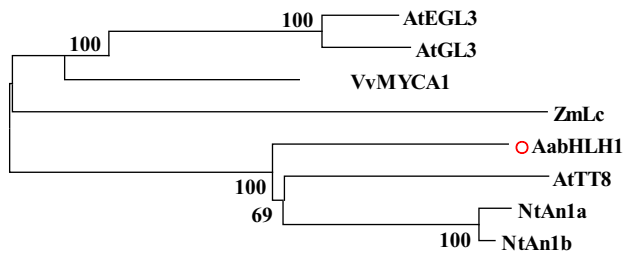

0.1

Fig. 1 Sequence alignments of AaMYB3, phylogenic tree of AaMYB3 and AabHLH1 with known R2R3-MYBs and bHLHs involved in proanthocyanidin (PA) biosynthesis. a Alignment of the full-length deduced amino acid sequence of AaMYB3 with the other R2R3-MYBs. The R2 and R3 domains are shown; the bHLH interacting motif are indicated in the red box; the conserved motifs VI[R/P]TKAx1RC[S/T] for the PA-regulating MYBs PA-clade 2 are indicated in the green box, and the conserved motifs $K[I / N] \times 2 P K P \times 1 R \times 2 S[/ / L]$ for PA-clade 1 are indicated in the blue box. Identical nucleotides are shown on a black background, and gaps are indicated by dashes. b Phylogenetic relationship of AaMYB3 with selected known PA and anthocyanin MYB regulators from anthurium and other species. The scale bar represents 0.1 substitutions per site. $\mathbf{c}$ Phylogenetic relationship of AabHLH1 with selected known bHLH regulators involved in PA and anthocyanin biosynthesis and trichome development from other species. The scale bar represents 0.1 substitutions per site. The MYB names and GenBank accession numbers are as follows: AaMYB3, MH349476, AaMYB1, AAO92352.1, AaMYB2, AML84515 (Anthurium andraeanum); PtMYB134, ACR83705.1, PtMYB115, XM 002302608.2 (Populus spp.);VVMYBPA1, CAJ90831.1, VvMYBPA2, ACK56131.1, VvMYBA1, BAD18977.1 (Vitis vinifera): AtTT2, CAC40021.1, AtMYB75, NP 176057.1 (Arabidopsis thaliana); DkMyb2, AB503699, DkMyb4, AB503701 (Diospyros kaki); ZmC1, 1613412E (Zea mays); bHLH names and GenBank accession numbers are as follows: AabHLH1, MH349477; AtEGL3, AEE3412, AtGLABRA3, AED94664.1, AtTT8, AEE82802.1 (Arabidopsis thaliana); VvMYCA1, NP 001267954.1 (Vitis vinifera); ZmLc, NP 001105339.1 (Zea mays); NtAn1a, AEE99257.1, NtAn1b, and AEE99258.1 (Nicotiana tabacum)

$\mathrm{Nr}$ and Swiss-Prot protein databases (data not shown). Among these, one unigene with a length of $1042 \mathrm{bp}$ containing an 891-bp ORF matched AtTT2, which is specific to the PA pathways in Arabidopsis, and one unigene with a length of 2694 bp containing a 2115-bp ORF matched a bHLH activator of PA synthesis in Arabidopsis, AtTT8. Therefore, these two candidate proteins may play roles in the modulation of PA biosynthesis in anthurium. The ORFs of the MYB and bHLH TF genes were amplified using RT-PCR from the spathe of the cultivar "Tropical" sampled at developmental stage 2. The two genes were designated $A a M Y B 3$ and AabHLH1, respectively, and encoded proteins of 297 and 705 amino acids, respectively.
An analysis of the deduced amino acid sequence of AaMYB3 suggested that an N-terminal R2R3 repeat corresponds to the DNA-binding (MYB) domain. Similarly to other related MYBs, the bHLH interaction motif [D/E] $\mathrm{Lx} 2[\mathrm{R} / \mathrm{K}] \mathrm{x} 3 \mathrm{~L} \mathrm{x} 6 \mathrm{Lx} 3 \mathrm{R}$ was identified in the highly conserved N-terminal R2R3 region of AaMYB3. In an alignment analysis with MYBs known to function in the regulation of $\mathrm{PA}$ biosynthesis and an additional two anthurium anthocyanin MYB regulators, we identified the C-terminal motif conserved in the AtTT2-like MYBs, $\mathrm{VI}[\mathrm{R} / \mathrm{P}] \mathrm{TKAx}{ }_{1} \mathrm{RC}[\mathrm{S} / \mathrm{T}]$, in AaMYB3. That indicates that AaMYB3 is very closely related to VvMYBPA2, AtTT2, and DkMYB2 and belongs to the PA-clade 2 MYB regulators (Fig. 1a). The conversed motif K[I/V] 
$\mathrm{x} 2 \mathrm{PKPx} 1 \mathrm{Rx} 2 \mathrm{~S}[\mathrm{I} / \mathrm{L}]$ is only found in the PA-clade $1 \mathrm{MYBs}$, such as VvMYBPA1 and DkMYB4. The two PAregulating motifs are not identified in the other two anthurium anthocyanin regulatory MYBs identified, AaMYB1 and AaMYB2. In a phylogenetic analysis, AaMYB3 clustered with AtTT2, VvMYBPA2, and PtMYB134 in PA-clade 2, and AaMYB1 and AaMYB2 from anthurium clustered with the anthocyanin regulatory MYBs AtMYB75 and VvMYBA1 (Fig. 1b). These sequence and phylogenetic analyses suggest that AaMYB3 plays a role in PA biosynthesis.

A sequence analysis of AabHLH1 showed the following conserved motifs: the MYB interaction region at the N-terminal; the bHLH domain in the C-terminal region, and the transactivation (ACT) domain (Fig. S1). In a phylogenetic analysis, AabHLH1 clustered with AtTT8 and NtAn1a/NtAn1b, which are involved in the biosynthesis of flavonoids, such as PAs and anthocyanins (Fig. 1c). These results suggest that AabHLH1 participates in the biosynthesis of the PAs or other flavonoids in anthurium.

\section{AaMYB3 is predominately expressed in the spathe and negatively correlates with anthocyanin accumulation in the spathes of cultivars with different color phenotypes}

To explore the transcript profiles of the new MYB and bHLH genes, cDNA samples from different tissues of anthurium were analyzed using q-PCR. We detected the transcripts of $A a M Y B 3$ and $A a b H L H 1$ in various anthurium tissues with no obvious tissue specificity. The $A a M Y B 3$ transcripts were predominantly detected in the spathe, followed by the leaves and then the spadix (Fig. 2a). The order of the AabHLH1 expression from the highest to lowest was as follows: peduncle $>$ spathe $>$ mature leaf and young leaf $>$ spadix (Fig. 2b). Factoring in the PA and anthocyanin contents of various tissues, the relative expression levels of $A a M Y B 3$ were high in the spathes and leaves where PA primarily accumulates (Fig. 2c). The AaMYB3 expression is associated with anthocyanin accumulation in various "Tropical" tissues (Pearson Correlation Coefficient, $r=0.832^{* * *}, P<0.01$ ) (Fig. 2d). However, the expression pattern of AabHLH1 was not related to the accumulation pattern of the PA or the anthocyanin (Fig. 2c, d).

To further investigate the correlation between the expression of AaMYB3 and AabHLH1 and the amounts of flavonoid present, the expression levels of the two TF genes and the PA and anthocyanin contents were determined in spathes with red, pink, purple, pale-pink, white, and green phenotypes. AaMYB3 and AabHLH1 were expressed in the spathes of all the cultivars tested where PAs also accumulated (Fig. 2e-g). Notably, anthocyanins accumulated primarily in the red spathe (Tropical) and then in the purple (Rapido) and pink spathes (Pink Champion) (Fig. 2h). These results further suggest that the expression of AaMYB3 is negatively related to anthocyanin accumulation in the spathe of anthurium cultivars with various color phenotypes $\left(r=-0.669^{* m * n}, p<0.01\right)$.

\section{Complementation of the Arabidopsis tt8 and tt2 mutants by the ectopic expression of AabHLH1 and AaMYB3}

To test whether AabHLH1 and AaMYB3 function as PA regulators, the two genes were introduced into the $t t 8$ and $t t 2$ mutants, respectively, and expressed under the control of the cauliflower mosaic virus 35S (CaMV35S) promoters (Fig. 3a). The transgenic $\mathrm{T}_{3}$ seeds of the AabHLH1-tt8 line had a brown seed coat, which was stained black by the DMACA reagent such as the wild type (Fig. 3b). This result demonstrates that the overexpression of AabHLH1 complemented the $t t 8$ mutant seed coat phenotype. The color of the $\mathrm{T}_{3}$ seed coat in the AaMYB3-tt2 line was slightly darker than that of the $t t 2$ mutant before and after DMACA staining (Fig. 3c). Although the restoration of the seed coat phenotype by $A a M Y B 3$ complementation was not as obvious as during the AabHLH1 complementation in the $t t 8$ mutant, the expression of the PA-specific enzyme gene $A t A N R$ was clearly upregulated in all the transgenic AaMYB3-tt2 lines (Fig. S2).

\section{Effects of $A a M Y B 3$ and $A a b H L H 1$ overexpression in tobacco}

To investigate whether the ectopic expression of AaMYB3 and AabHLH1 affects PA biosynthesis, we analyzed transgenic tobacco plants that independently overexpressed $A a M Y B 3$ or $A a b H L H 1$ and simultaneously overexpressed both AaMYB3 and AabHLH1 (AaMYB3 + AabHLH1). Two independent $\mathrm{T}_{1}$ transgenic lines from each construct were selected based on their levels of transgene expression (Fig. 4b). Under the same growing conditions, the corolla limb colors of the $\mathrm{T}_{1}$ AaMYB3overexpressing (ox) lines changed to light pink compared with the control plants transformed with the empty vector (EV). The corolla limb color of the $\mathrm{T}_{1}$ AabHLH1-ox lines showed no obvious phenotypic changes relative to the EV control. Interestingly, the corolla limbs of the $\mathrm{T}_{1}$ $A a M Y B 3+A a b H L H 1$-ox plants were much paler pink than either the AaMYB3-ox plants or the EV control plants (Fig. 4a). An analysis of the anthocyanin contents showed changes that paralleled those in the color phenotype. The levels of total anthocyanins from the lowest to highest were as follows: $A a M Y B 3+A a b H L H 1$-ox lines $<A a M Y B 3$-ox lines $<\mathrm{EV}$ or AabHLH1-ox lines. The PA contents in the tobacco corolla limbs correlated negatively with the anthocyanin content. The AaMYB3 + AabHLH1-ox lines showed the highest PA content, and the AaMYB3-ox lines also showed markedly higher PA content in the corolla limbs than the EV or AabHLH1-ox 

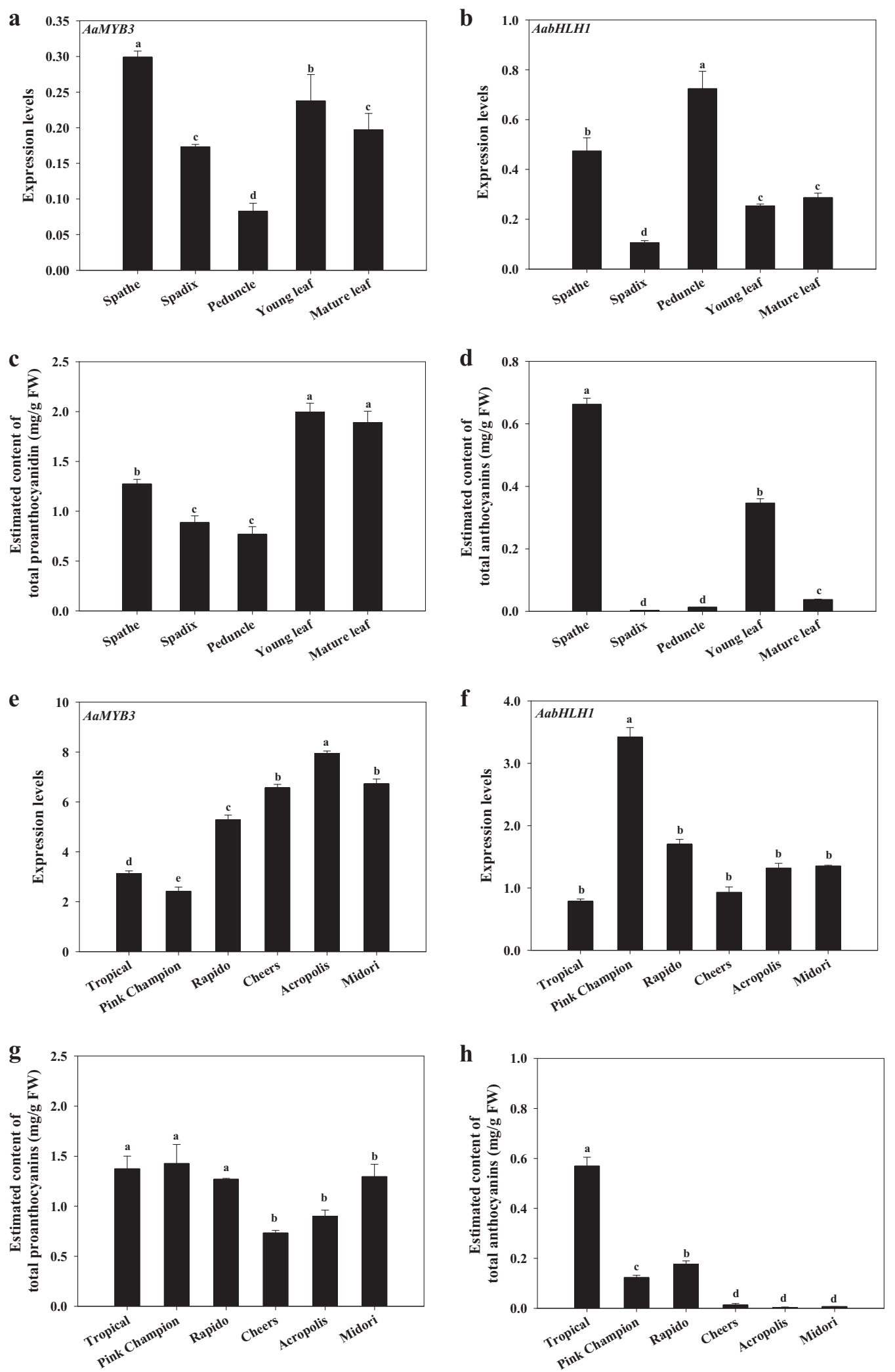

Fig. 2 The expression pattern of $A a M Y B 3$ and $A a b H L H 1$, as well as the content of total proanthocyanidins and anthocyanins, in anthurium. The expression of AaMYB3 (a) and AabHLH1 (b), the content of total proanthocyanidins (c) and anthocyanins (d) in various tissues of the cultivar "Tropical"; The expression level of AaMYB3 (e) and AabHLH1 (f), the content of total proanthocyanidins ( $(\mathbf{g})$ and anthocyanins (h) in the spathes of different cultivars with various color phenotypes. The data were presented as the mean \pm SD $(n=3)$. Values with different letters are significantly different according to Duncan's multiple range tests at the $5 \%$ level 


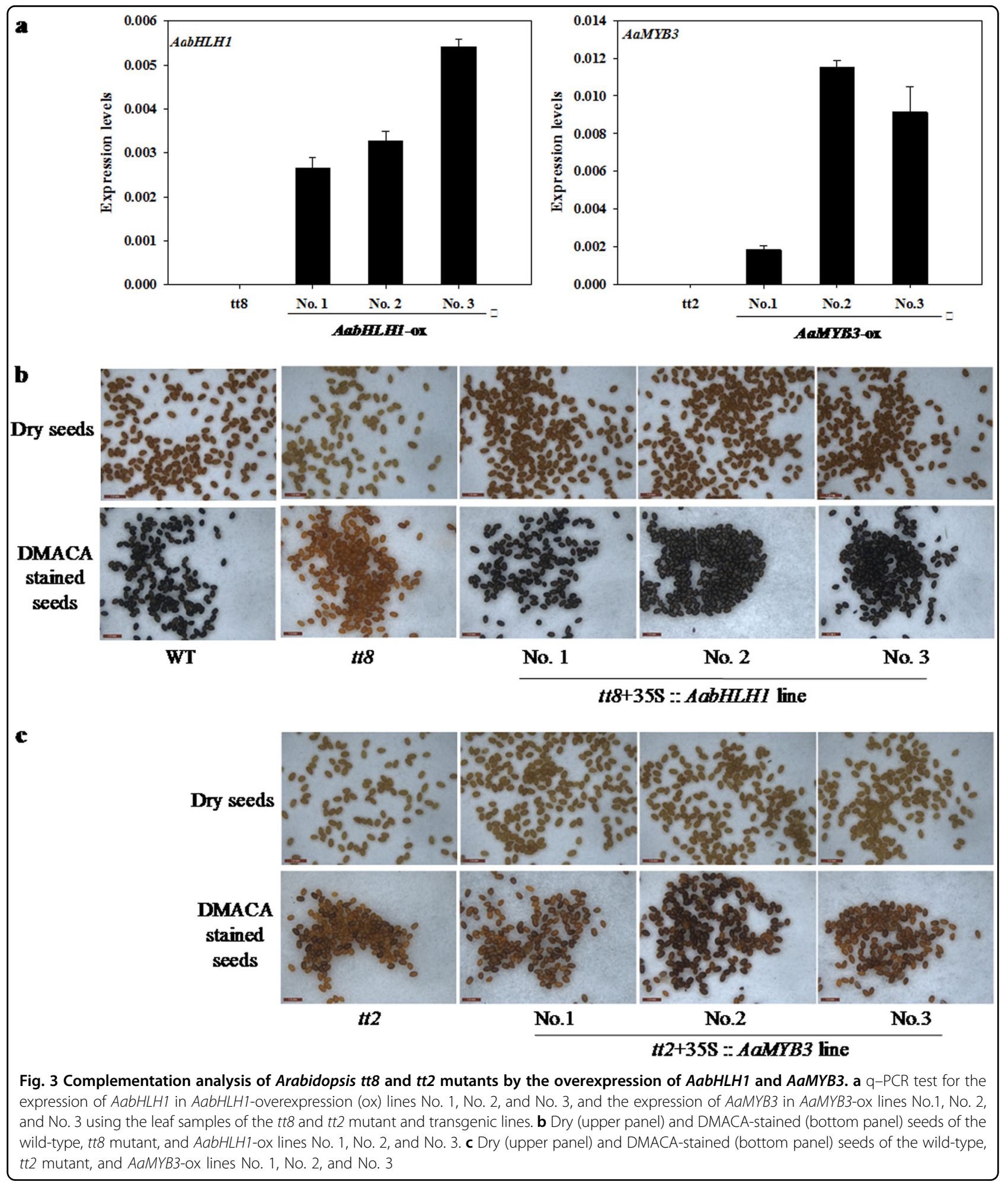

lines (Fig. 4c). The total flavonoid and polyphenol contents were also measured. Only the AaMYB3+ AabHLH1-ox lines showed higher total flavonoid and polyphenol contents than the other lines (Fig. 4d). These results suggest that the overexpression of AaMYB3 significantly enhanced the accumulation of the PAs leading to a pale pigmentation in the corolla limb of the transgenic plants. The PA regulatory function of AaMYB3 was increased synergistically by AabHLH1. Simple AabHLH1 overexpression had no significant effect on the 

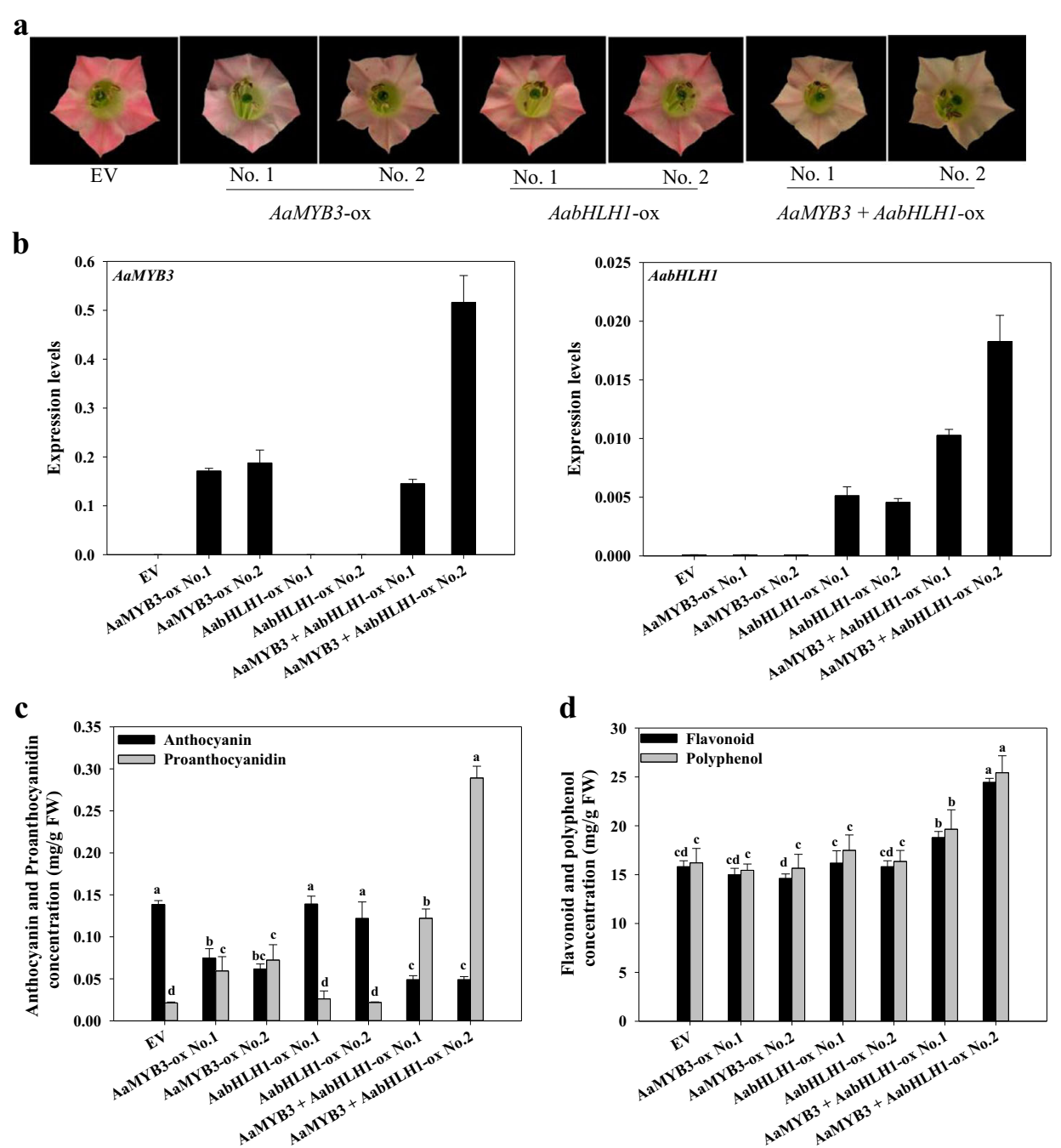

e
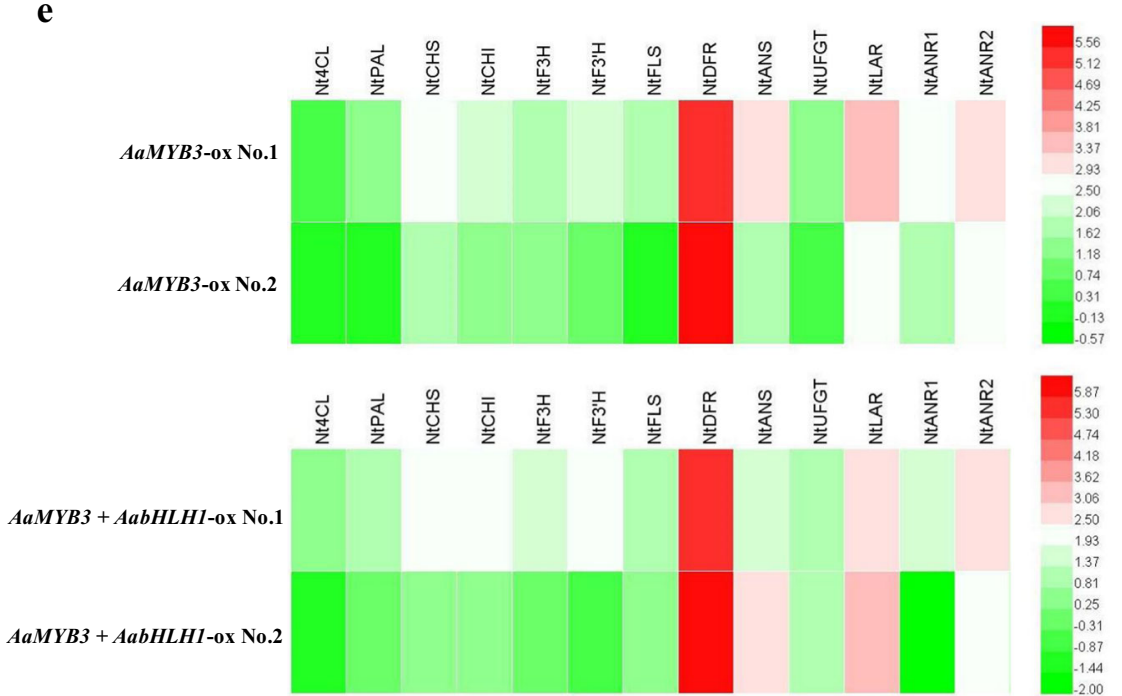

Fig. 4 (See legend on next page.) 
(see figure on previous page)

Fig. 4 Typical phenotypes, secondary metabolite amounts, and gene expression of the transgenic tobacco corolla limbs. a Typical floral phenotypes of the EV (empty vector transformed line), AaMYB3-overexpression (ox) lines No. 1 and No. 2, AabHLH1-ox lines No. 1 and No. 2, and $A a M Y B 3+$ AabHLH1-ox lines No. 1 and No. 2. b AaMYB3 and AabHLH1 expression in the EV and transgenic tobacco corolla limbs tested using q-PCR. c Total anthocyanin and proanthocyanidin contents in the corolla limbs of the EV and transgenic tobacco lines. $\mathbf{d}$ Total flavonoid and polyphenol contents in the corolla limbs of the EV and transgenic tobacco lines. e The relative expression analysis of the flavonoid biosynthetic genes in the tobacco corolla limbs of AaMYB3-ox lines and AaMYB3 + AabHLH1-ox lines. Color bar: Log 2 (fold changes). The data are presented as the mean \pm SD $(n=3)$. Values with different letters are significantly different according to Duncan's multiple range tests at the $5 \%$ level
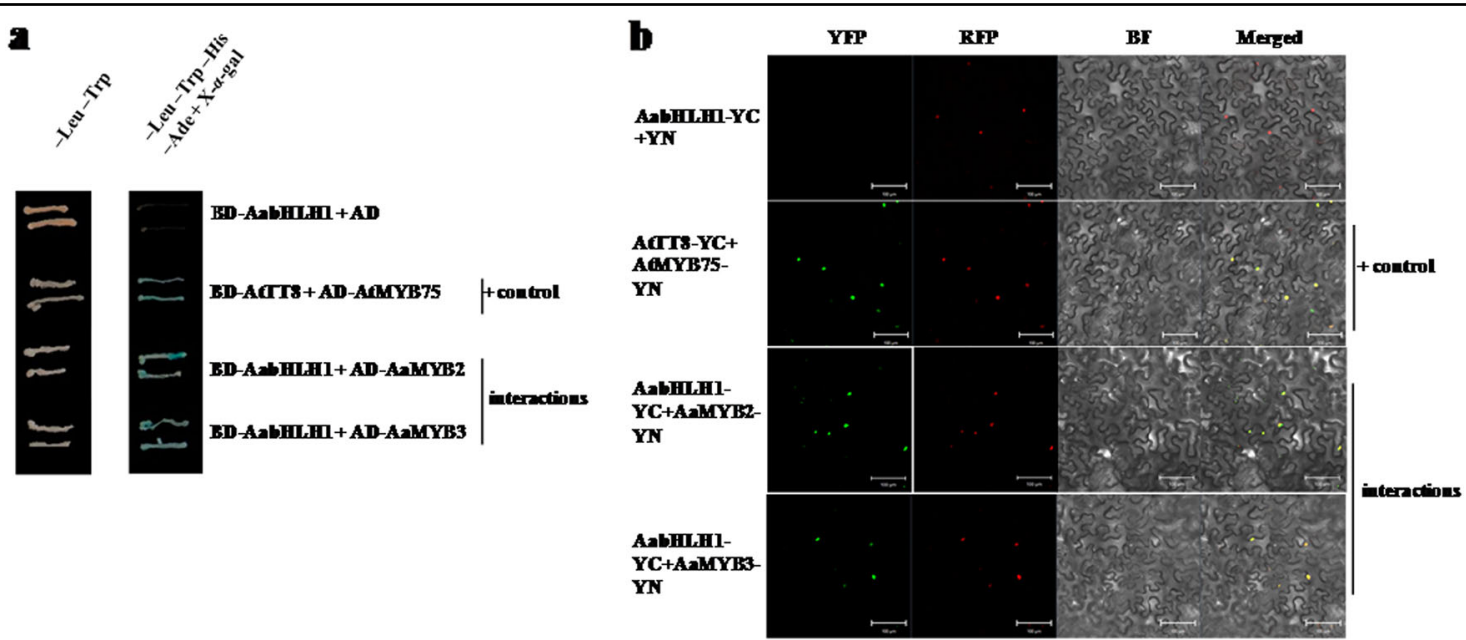

Fig. 5 Interactions between AabHLH1 and AaMYB2 and AaMYB3 detected using the Y2H and BiFC assays. a Y2HGold yeast cells containing plasmids pGADT7 + pGBK-AabHLH1, pGAD-AaMYB2 + pGBK-AabHLH1 or pGAD-AaMYB3 + pGBK-AabHLH1 were grown on double- and quadrupleselection media, and pGAD-AtMYB75 + pGBK-AtTT8 was used as the positive control. The X-a-gal assay was performed to confirm the positive interactions. b Bimolecular fluorescence complementation visualization of the AabHLH1 and AaMYB2 and AaMYB3 interaction in the N. benthamiana leaf epidermal cells. The AtMYB75-AtTT8 interaction was used as a positive control. YFP yellow fluorescent protein field, RFP red fluorescent protein, which indicated the nuclear localization of Arabidopsis SR45; BF bright field. Merged, overlay of the YFP, RFP, and BF field. Bars, $100 \mu m$

anthocyanin or PA in the transgenic tobacco corolla limbs.

A q-PCR analysis was performed to further analyze the effects of the ectopic expression of $A a M Y B 3$ and $A a M Y B 3+A a b H L H 1$ on the target enzyme genes involved in the biosynthesis of flavonoids in tobacco. The overexpression of $A a M Y B 3$ alone affected the expression of the anthocyanin- and PA-related genes in the transgenic lines, and in particular, clearly upregulated the expression of the late anthocyanin enzyme genes NtDFR and NtANS and the key PA biosynthetic genes $N t L A R$ and NtANR (Fig. 4e). In the AaMYB3+ $A a b H L H 1$-ox lines, the expression pattern of the genes in the flavonoid biosynthesis pathway was similar to that in the AaMYB3-ox lines (Fig. 4e). These data indicate that AaMYB3 alone or together with AabHLH1 upregulates or activates the expression of key anthocyanin and PA biosynthetic key genes, ultimately promoting PA accumulation in transgenic tobacco.

\section{Interaction of AabHLH1 with different AaMYBs partners}

The MBW ternary transcription complex is usually formed to regulate flavonoid biosynthesis in plants. The interaction of AabHLH1 with AaMYB3 and the previously reported anthocyanin regulator AaMYB2 was investigated using a $\mathrm{Y} 2 \mathrm{H}$ assay. The autoactivation of pGBK-AaMYB3 or pGBK-AaMYB2 was observed on SD/ -Trp/-His/-Ade media containing 5-bromo-4-chloro-3indolyl- $\alpha$-D-galactopyranoside (X- $\alpha$-Gal). Therefore, $A a M Y B 3$ or $A a M Y B 2$ was fused to the GAL4 DNAactivating domain, and $A a b H L H 1$ was fused to the GAL4 DNA-binding domain. As shown in Fig. 5a, the protein-protein interactions between AabHLH1 and AaMYB3 or AaMYB2 were demonstrated by the growth of colonies containing both the pGAD-AaMYB3 and pGBKAabHLH1 vectors or both the pGAD-AaMYB2 and pGBK-AabHLH1 vectors on SD/-Leu/-Trp/-His/-Ade $+\mathrm{X}-\alpha$-Gal media. Thus, the $\mathrm{Y} 2 \mathrm{H}$ assay suggested that AabHLH1 interacts with both AaMYBs and forms a transcriptional complex. 
The interaction of AabHLH1 with AaMYBs was confirmed using a BiFC assay. AabHLH1 tagged with the split cYFP fragment (YC) and AaMYB3 or AaMYB2 tagged with the split nYFP fragment (YN) were transiently coexpressed in $N$. benthamiana leaves. As shown in Fig. 5b, the YFP fluorescent signal was detected in tobacco epidermal cells expressing both the AaMYB3-YN and AabHLH1-YC fusion proteins and the AaMYB2-YN and AabHLH1-YC fusion proteins, and they successfully merged with the RFP fluorescent signals, while no YFP fluorescent signal was detected in the epidermal cells expressing AabHLH1-YC with only YN. The BiFC assay demonstrated the interaction between AabHLH1 and AaMYB2 and AaMYB3 in vivo, and their protein-protein complexes were localized in the nucleus.

\section{Expression trends in $A a M Y B 3$ and $A a b H L H 1$ are coincident with those of several anthocyanin and PA biosynthetic genes}

The ectopic expression $A a M Y B 3$ alone or the coexpression of $A a M Y B 3$ and $A a b H L H 1$ in tobacco stimulated PA production by activating a number of anthocyanin and PA biosynthetic genes. Therefore, AaMYB3 and AabHLH1 are hypothesized to act as PA activators in anthurium. To investigate the possible regulatory roles of AaMYB3 and AabHLH1, a gene coexpression analysis was performed in the developing spathes and spadices of the cultivars "Vitara" and "Tropical", because they are the primary ornamental organs, and AaMYB3 is predominantly expressed in the spathe. The spathes of both cultivars simultaneously contained anthocyanins and PAs in all the developmental stages investigated (Fig. 6a, b). As shown in Fig. 6a, the expression of AaMYB3 decreased progressively during development in the spathe of the cultivar "Vitara", and the expression of AabHLH1 decreased in stage 2 and then increased again and peaked in stage 5. The expression pattern of $A a M Y B 3$ appeared to be related to PA accumulation $\left(r=0.840^{* * *}, p<0.01\right)$ and negatively related to anthocyanin accumulation $\left(r=-0.741^{* * *}, p<0.01\right)$ (Fig. 6a). Of the anthocyanin and PA biosynthetic genes, the early flavonoid synthetic genes $A a C H S$ and $A a F 3 H$, the anthocyanin-specific genes $A a D F R$ and AaANS and the PA-specific genes AaLAR and $A a A N R$ were coordinately expressed with $A a M Y B 3$ (Fig. 6a). The correlation coefficients are shown in Table S2. However, unlike AaMYB3, the expression of AabHLH1 showed no obvious relationship with either PA or anthocyanin accumulation and was only strongly coexpressed with $A a F 3^{\prime} H\left(r=0.952^{* * *}, p<0.01\right)$. In the spathe of the cultivar "Tropical", the expression of $A a M Y B 3$ peaked at stage 2 before subsequently decreasing, reaching its lowest level in stage 4 and then increased again in stage 5 (Fig. 6b). The expression of AabHLH1 increased gradually and peaked in stage 4 after which it decreased slightly (Fig. 6b). The expression patterns of $A a M Y B 3$ and AabHLH1 in the 'Tropical' spathe were not associated with anthocyanin or PA accumulation (Fig. 6a, b). The correlation analysis suggests that $A a M Y B 3$ coexpressed with $A a D F R, A a L A R$, and $A a A N R$ and that $A a b H L H 1$ is coexpressed with $A a C H S, A a F 3 H$, $A a F 3^{\prime} H$, and $A a A N S$ in the "Tropical" spathe (Fig. 6b, Table S2).

The spadix of cultivar "Vitara" accumulated both anthocyanins and PAs at all the development stages investigated (Fig. S3a), while the "Tropical" spadix accumulated only PAs during its development (Fig. S3b). The expression pattern of $A a M Y B 3$ showed no obvious correlation with either anthocyanin or PA accumulation in the spadix of "Vitara" (Fig. S3a). Similar to that in the spathe, AaMYB3 was coexpressed with most of the genes in the anthocyanin and PA biosynthetic pathways, such as AaCHS, AaCHI, AaF3H, AaDFR, AaANS, and AaLAR (Fig. S3a, Table S2). AabHLH1 expression positively correlated with that of AaUFGT and AaANR (Fig. S3a, Table S2). In the "Tropical" spadix, AaCHS, $A a F 3 H$, AaDFR, AaANS, AaLAR, and AaANR showed a similar expression pattern to that of $A a M Y B 3$, and $A a C H I, A a F 3^{\prime}$ $H$, and AaUFGT were coexpressed with AabHLH1 (Fig. S3b). These results suggest that AaMYB3 may target several genes in the anthocyanin and PA biosynthetic pathways and activate PA biosynthesis in anthurium. AabHLH1 may play a role in the whole flavonoid biosynthetic pathway and is not specific to PA biosynthesis.

\section{Discussion}

PAs are colorless flavonoid polymers that accumulate in plants and are usually controlled by the MBW TF complex $^{5}$. In this study, we report the isolation and analysis of the R2R3-MYB gene AaMYB3 and bHLH TF gene AabHLH1 from anthurium. The AaMYB3 protein is similar to the known PA regulators grape VvMYBPA2, Arabidopsis AtTT2, poplar PtMYB134, and persimmon DkMyb2, which share a short conserved VI[R/P] $\mathrm{TKAx}_{1} \mathrm{RC}[\mathrm{S} / \mathrm{T}]$ motif $^{10}$. According to Zhu et al. ${ }^{21}$, MYB $\mathrm{PA}$ regulators can be categorized into two subgroups: PAclade 1 includes VvMYBPA1 and DkMyb4, and PA-clade 2 includes AtTT2, VvMYB2, PtMYB134, and DkMyb2. The two types of PA regulators display different expression patterns and recognize different types of motifs in the promoter regions of the PA pathway genes ${ }^{21}$. According to our phylogenetic analysis, AaMYB3 clustered in the PA-clade 2 subgroup ${ }^{21}$, clearly distinct from the previously reported anthocyanin regulators $\mathrm{AaMYB1}^{27}$ and AaMYB2 ${ }^{29}$. AabHLH1 is homologous with Arabidopsis AtTT8 and tobacco NtAn1, which are members of the bHLH TF subgroup IIIf, which is involved in the regulation of PA and flavonoid biosynthesis ${ }^{8,49,50}$. Our amino acid sequence analysis suggested that AaMYB3 contains 

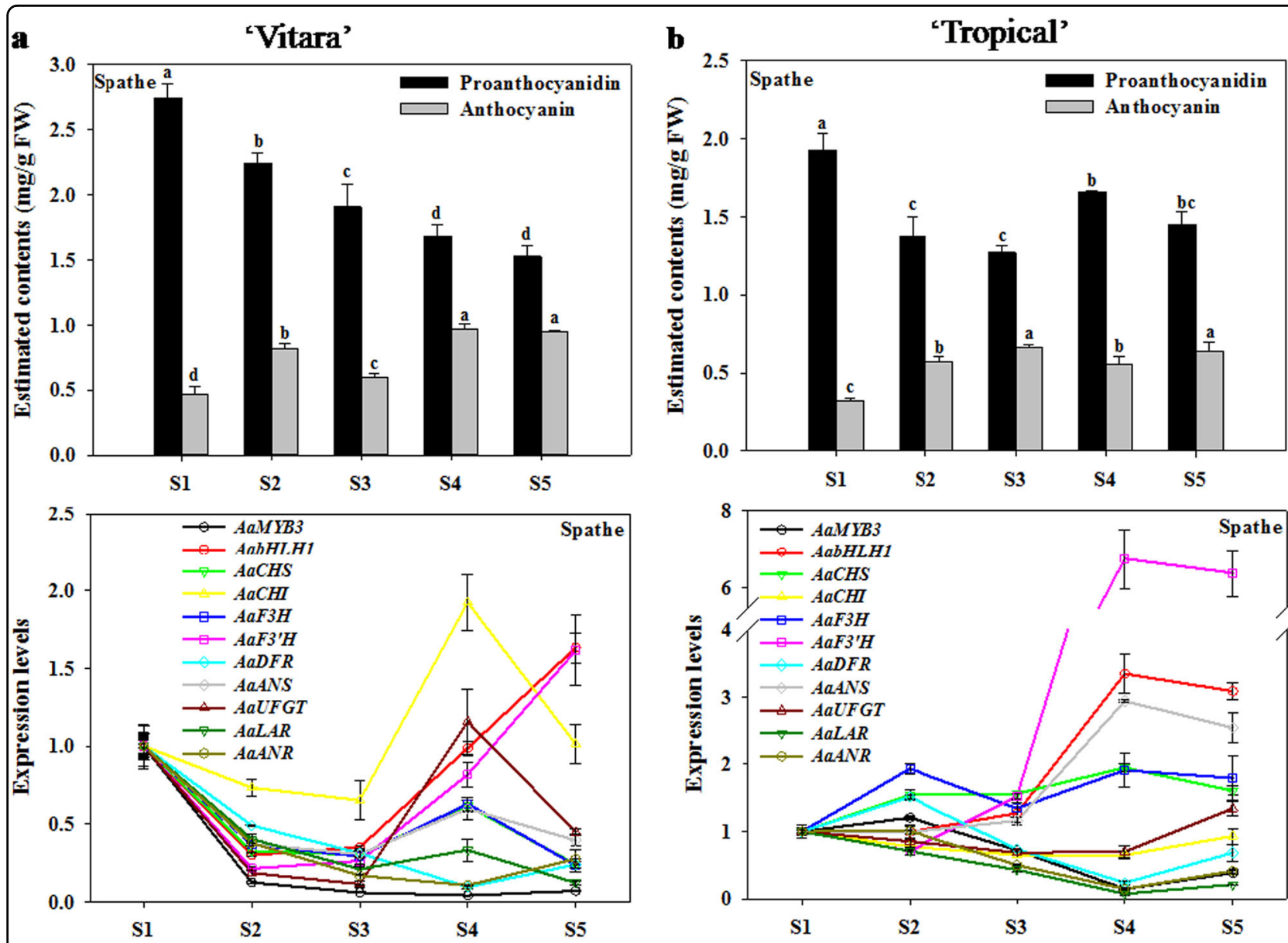

$\mathbf{c}$

4-Coumaroyl-CoA 3 Molonyl-CoA

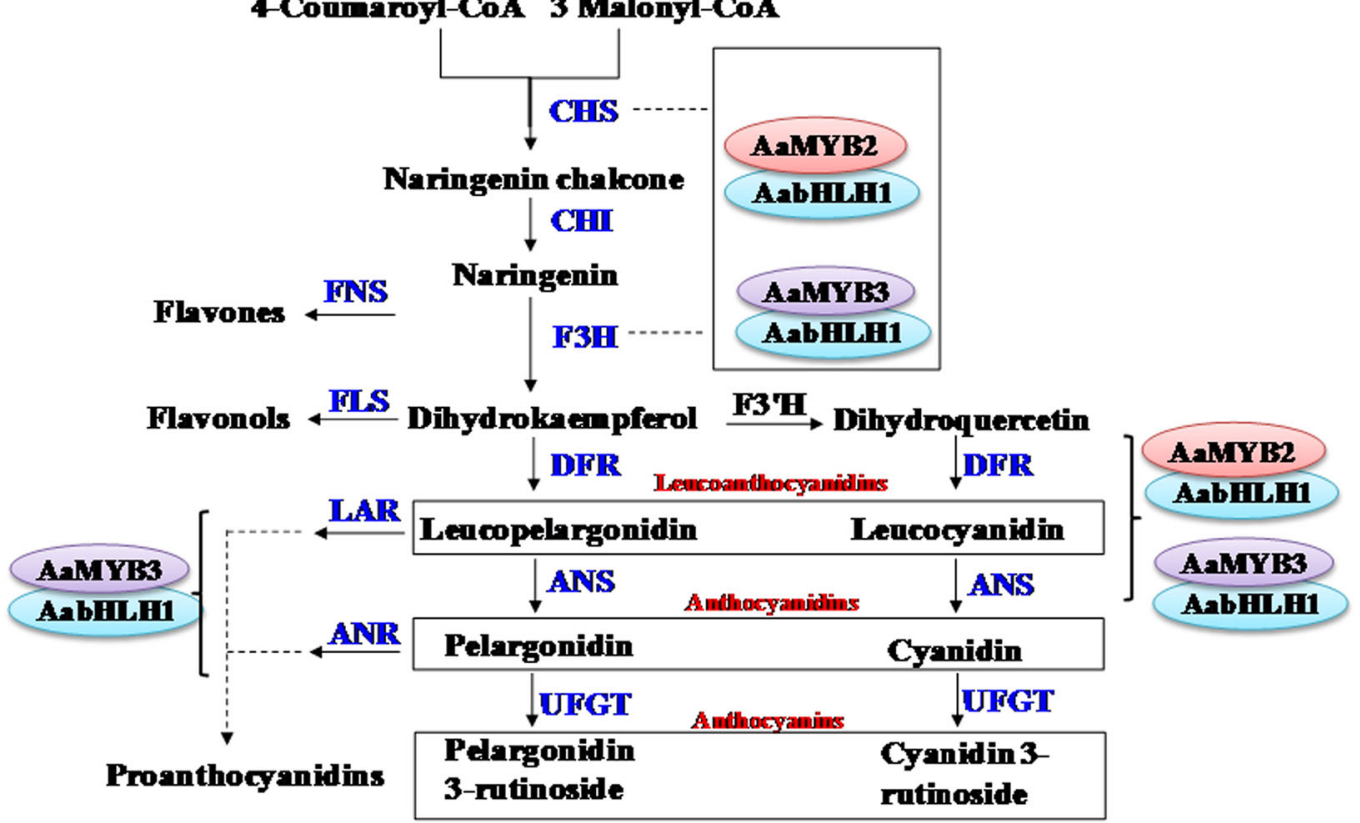

Fig. 6 Analysis of the function of AaMYB3 and AabHLH1 in anthurium. The detection for the content of total proanthocyanidins and anthocyanins, as well as the expression of AaMYB3, AabHLH1, and the flavonoid biosynthetic genes in the developmental spathe of cultivars "Vitara" (a) and "Tropical" (b). The data were presented as the mean \pm SD $(n=3)$. Values with different letters are significantly different according to Duncan's multiple range tests at the $5 \%$ level. c A proposed model for the regulation of proanthocyanin and anthocyanin biosynthesis by AaMYB2, AaMYB3, and $\mathrm{AabHLH} 1$ in the anthurium spathe 
the bHLH interaction motif and that AabHLH1 contains the MYB interaction region. Therefore, it is possible that the two proteins physically interact. These results imply that these two TF are involved in the PA biosynthetic pathway.

In a previous study, we showed that the expression pattern of $A a M Y B 2$ correlates strongly with anthocyanin accumulation ${ }^{29}$. The expression pattern of AaMYB3 in the different tissues of anthurium cultivar "Tropical" was similar to that of the anthocyanin regulator gene $A a M Y B 2$, which was predominantly expressed in the spathe and exhibited a positive correlation with anthocyanin accumulation. However, unlike $A a M Y B 2$, the expression of $A a M Y B 3$ exhibited a negative correlation with anthocyanin accumulation in the spathes of different anthurium cultivars with various color phenotypes. It should be noted that AaMYB3 is more strongly expressed in the green and white spathes where proanthocyanin accumulates, and no anthocyanin was detected. This indicated that the expression pattern of AaMYB3 in anthurium may be associated with PA accumulation. This was confirmed in the developing "Vitara" spathes in which the expression pattern of AaMYB3 was exactly consistent with the accumulation of PA and correlated negatively with anthocyanin accumulation. Similar results have been described for PA regulators in grapevine $\left(\mathrm{VvMYBPA}^{13}{ }^{13}\right.$ and coleus $\left(\mathrm{SsMYB3}^{21}\right)$.

Because AaMYB3 is homologous to AtTT2, a complementation test of the Arabidopsis tt 2 mutant was used to functionally characterize AaMYB3. In the Arabidopsis $t t 2$ and $t t 8$ mutants, the AtTT2-AtTT8-AtTTG1 ternary transcription complex fails to form, and the expression of the PA biosynthesis gene AtANR is not induced ${ }^{8}$. The lack of PAs in the two mutants causes a yellow seed phenotype, and the overexpression of a homologous gene restored the seed color. Although AaMYB3 clustered in PA-clade 2, the overexpression of AaMYB3 caused only a slight recovery of the $t t 2$ mutant seed coat phenotype. This was not as marked as the effects of other PA-clade 2 MYBs, such as AtTT2 ${ }^{10}$ and VvMYBPA1 ${ }^{13}$. To analyze the function of AaMYB3 in the $t t 2$ mutant, the expression of the genes in the PA pathway was detected in the leaves of the $\mathrm{T}_{3}$ transgenic AaMYB3-tt2 plants. The expression of the PA-specific AtANR was significantly upregulated compared with that in the $t t 2$ mutant control. This implied that AaMYB3 activates the expression of AtANR to some degree. However, this degree of activation is insufficient to cause enough PA accumulation in the seed coat and therefore, to completely complement the mutant phenotype. In the transcriptional regulation of PA biosynthesis in Arabidopsis, TT2 is responsible for the specific recognition of the promoter of $A t A N R$ in combination with $\mathrm{TT}^{8}$. In the AaMYB3-tt2 transgenic line, AaMYB3 might not have combined well with AtTT8 to efficiently activate PA biosynthesis. The function of AaMYB3 was also characterized by its ectopic expression in tobacco. The overexpression of $A a M Y B 3$ alone produced light pink corolla limbs with greatly accumulated PAs. This is consistent with the overexpression of other PA-related MYB regulators in tobacco, coleus $\mathrm{SsMYB}^{21}$, grapevine $\mathrm{VvMYBPA}^{51}$, and Malus crabapple McMYB12b ${ }^{22}$. The functions of these MYBs are thought to be conserved across a diverse range of species. The overexpression of $A a M Y B 3$ in the corolla limbs of transgenic tobacco caused the significantly upregulated expression of the key genes in the anthocyanin (NtDFR and NtANS) and PA biosynthetic pathways (NtLAR and NtANR). This result is similar to the results when $S s M Y B 3^{21}$ and $V v M Y B P A 1^{51}$ were overexpressed in the transgenic tobacco flowers and shows that AaMYB3 is functionally homologous to the MYBs described above, which are involved in the regulation of PA biosynthesis.

The expression pattern of $A a b H L H 1$ was not associated with either PA or anthocyanin in various anthurium tissues, the spathes of different cultivars, or at different developmental stages of the spathe. This is consistent with the expression of the anthocyanin-modulating PabHLH3 in sweet cherry ${ }^{52}$ but differs from the bHLHTF-controlled anthocyanin biosynthesis in other horticultural plants, such as LcbHLHs in litchi ${ }^{53}$ and DhbHLH1 in dendrobium ${ }^{44}$. It can be hypothesized that the expression of $A a b H L H 1$ in anthurium is not directly involved in the accumulation of the PA or anthocyanin pigments. Our phylogenetic analysis indicates that AabHLH1 is homologous to AaTT8. Therefore, a complementation test of the Arabidopsis tt 8 mutant was performed to functionally characterize AabHLH1. As expected, the overexpression of AabHLH1 in the $t t 8$ mutant complemented the seed coat phenotype. In the AabHLH1-tt8 transgenic line, AabHLH1 functioned as AtTT8, combined with AtTT2 and successfully activating the PA pathway. This suggests that AabHLH1 plays a role in PA biosynthesis. When AabHLH1 was overexpressed in tobacco, it caused no significant change in the phenotype or in the accumulation of the anthocyanins or PAs in the corolla limbs. This outcome is similar to the phenomenon observed when tobacco was transformed with the MrbHLH1 of Chinese bayberry. The transgenic tobacco line overexpressing MrbHLH1 showed a phenotype similar to that of the control ${ }^{54}$. However, the line overexpressing both MrbHLH1 and MrMYB1 accumulated significant amounts of anthocyanin in the whole plant ${ }^{54}$. Therefore, we hypothesize that AabHLH1 requires an appropriate MYB partner to participate in PA biosynthesis. A transgenic tobacco line overexpressing both AabHLH1 and AaMYB3 was obtained by crossing transgenic plants that separately 
overexpressed $A a M Y B 3$ and $A a b H L H 1$ as described by Xie et al. ${ }^{55}$. The offspring of the cross, which coexpressed $A a M Y B 3$ and AabHLH1, had much paler pink corolla limbs than the AaMYB3-ox line, and they accumulated PAs strongly, as well as total flavonoids and polyphenols. As in the AaMYB3-ox line, the tobacco anthocyanin and PA biosynthetic genes NtDFR, NtANS, NtLAR, and $N t A N R$ were strongly upregulated in the AaMYB3+ AabHLH1-ox line. As a result, in the transgenic tobacco, AabHLH1 recognized AaMYB3 as its partner and enhanced the expression of $A a M Y B 3$ for efficient PA biosynthesis. The formation of the AaMYB3-AabHLH1 and AaMYB2-AabHLH1 protein complex was confirmed using the $\mathrm{Y} 2 \mathrm{H}$ and BiFC assays. These provided further evidence of the involvement of AabHLH1 in PA biosynthesis via its physical interaction with AaMYB3. There was evidence that AabHLH1 interacts with AaMYB2, which was identified as an anthocyanin regulator in anthurium in our previous study ${ }^{29}$. Therefore, AabHLH1 may also play a role in anthocyanin biosynthesis.

The PA regulatory functions of AaMYB3 and AabHLH1 were characterized by their exogenous expression in Arabidopsis and tobacco. However, the regulatory mechanism controlling PA production in anthurium is still unclear. Because AaMYB3 is primarily expressed in the spathe, our gene expression analysis was primarily performed in spathe tissues. In the red spathes of cultivars "Vitara" and "Tropical", the expression pattern of AaMYB3 was similar to that of AaCHS $\left(r=0.845^{* *}\right.$, $p<0.01), \quad A a D F R \quad\left(r=0.924^{* * *}, \quad p<0.01\right), \quad A a F 3 H$ $\left(r=0.842^{* * *}, \quad p<0.01\right)$, AaANS $\left(r=0.623^{* * n}, \quad p<0.01\right)$, $\operatorname{AaLAR}\left(r=0.904^{* * *}, p<0.01\right)$, and AaANR $\left(r=0.660^{* * *}\right.$, $p<0.01)$ and correlated well with proanthocyanin accumulation $\left(r=0.754^{* * *}, \quad p<0.01\right)$. This implies that AaMYB3 regulates the early biosynthetic genes in the flavonoid pathway ( $A a C H S$ and $A a F 3 H$ ) and the late biosynthetic genes AaDFR and AaANS, as well as the PAspecific genes AaLAR and AaANR. Similarly, the persimmon PA regulator DkMyb2 upregulated the expression of DkCHS, DkDFR, DkANS, DkLAR, and DkANR in transgenic persimmon calluses ${ }^{56}$. In contrast, the expression pattern of $A a b H L H 1$ was similar to that of $A a F 3^{\prime} H\left(r=0.645^{m * *}, P<0.01\right)$ in the spathes of the two cultivars. A previous study of the expression of the flavonoid pathway genes suggested that $A a F 3 H$ and $A a A N S$ are coregulated, while $A a D F R$ and $A a F 3^{\prime} H$ are separately regulated $^{23,26}$. Our previous study suggested that AaMYB2 regulates the expression of $A a F 3 H$ and AaANS, and possibly $A a C H S$, in the anthocyanin biosynthetic pathway of anthurium, while the expression pattern of $A a M Y B 2$ differed dramatically from that of $A a D F R$ and $A a F 3^{\prime} H^{29}$. In this study, a gene expression analysis suggested that $A a M Y B 3$ specifically coregulates AaDFR, $A a L A R$, and $A a A N R$ expression in the PA pathway, while
AabHLH1 is probably involved in the regulation of $A a F 3^{\prime}$ $H$. These results extend our understanding of the regulation of flavonoid biosynthesis in anthurium.

These results suggest that AaMYB3 functions as a negative regulator of anthocyanin accumulation and a positive regulator of PA accumulation in the anthurium spathe. This may occur by the upregulation of the expression of the genes for the enzymes specific to PAs biosynthesis, such as AaLAR and AaANR, and the acceleration of the reduction of leucocyanidin and anthocyanidin to promote the biosynthesis of PAs and repress the accumulation of anthocyanins. Based on the analyses described above, we propose a model of how anthocyanin and PA accumulation are affected by the anthocyanin regulator AaMYB2 and the PA regulator AaMYB3 in the anthurium spathe (Fig. 6c). In the red, pink, and purple spathes in which anthocyanin and PA accumulate simultaneously, both $A a M Y B 2$ and $A a M Y B 3$ are expressed, and the two TFs coregulate the expression of $A a C H S$, $A a F 3 H$, and AaANS. AaMYB3 specifically actives AaDFR, $A a L A R$, and AaANR expression. Therefore, anthocyanins and PAs accumulate together in the spathe. In the white and green spathes in which only PA accumulates, the expression of $A a M Y B 2$ is negligible, and AaMYB3 activates the PA biosynthetic pathway, causing the accumulation of PAs. AabHLH1 interacts with AaMYB3 and AaMYB2 in the transcriptional regulation process.

\footnotetext{
Acknowledgements

This research was supported financially by the Hainan Provincial Natural Science Foundation of China (No. 2018CXTD344) and the Central PublicInterest Scientific Institution Basal Research Fund for the Chinese Academy of Tropical Agricultural Sciences (Nos. 1630032017074 and 1630032017024).
}

\section{Author details}

${ }^{1}$ Tropical Crops Genetic Resources Institute, the Chinese Academy of Tropical Agricultural Sciences (CATAS) / Key Laboratory of Crop Gene Resources and Germplasm Enhancement in Southern China, Ministry of Agriculture, Danzhou 571737, China. ${ }^{2}$ The Engineering Technology Research Center of Tropical Ornamental Plant Germplasm Innovation and Utilization, Hainan Province, Danzhou 571737, China. ${ }^{3}$ Rubber Research Institute, CATAS/ Key Laboratory of Biology and Genetic Resources of Rubber Tree, Ministry of Agriculture, Danzhou 571737, China

\section{Author contributions}

L.C.H., Y.G.S., and Y.J.M. designed the research. L.C.H. performed the research. L.C.H. and Q.J. analyzed the data and wrote the manuscript. H.S.R. was responsible for the cultivation of the anthurium and transgenic tobacco plants. Y.G.S. and Y.J.M. edited the manuscript. All of the authors read and approved the final manuscript.

Conflict of interest

The authors declare that they have no conflict of interest.

\section{Publisher's note}

Springer Nature remains neutral with regard to jurisdictional claims in published maps and institutional affiliations.

Supplementary Information accompanies this paper at (https://doi.org/ 10.1038/s41438-018-0102-6). 
Received: 21 May 2018 Revised: 2 October 2018 Accepted: 11 October 2018 Published online: 01 January 2019

\section{References}

1. Dixon, R. A., Xie, D. Y. \& Sharma, S. B. Proanthocyanidins: a final fronter in flavonoid research? New Phytol. 165, 9-28 (2005).

2. Gonzalez de Colmenares, N. et al. Isolation, characterisation and determination of biological activity of coffee proanthocyanidins. J. Sci. Food Agric. 77, 368-372 (1998).

3. Barbehenn, R. V. \& Constabel, C. P. Tannins in plant-herbivore interactions. Phytochemistry 72, 1551-1565 (2011).

4. He, F., Pan, Q.-J., Shi, Y. \& Duan, C.-Q. Biosynthesis and genetic regulation of proanthocyanidins in plants. Molecules 13, 2674-2703 (2008).

5. Liu, J., Osbourn, A. \& Ma, P. Myb transcription factors as regulators of phenylpropanoid metabolism in plants. Mol. Plant 8, 689-708 (2015).

6. Xu, W., Lepiniec, L. \& Dubos, C. New insights toward the transcriptional engineering of proanthocyanidin biosynthesis. Plant Signal. Behav. 9, e28736 (2014).

7. Patra, B., Schluttenhofer, C., Wu, Y., Pattanaik, S. \& Yuan, L. Transcriptional regulation of secondary metabolite biosynthesis in plants. Biochim. Biophys. Acta 1829, 1236-1247 (2013).

8. Baudry, A., Heim, M. A., Dubreucq, B., Caboche, M., Weisshaar, B. \& Lepiniec, L. TT2, TT8, and TTG1 synergistically specify the expression of BANYULS and proanthocyanidin biosynthesis in Arabidopsis thaliana. Plant J. 39, 366-380 (2004).

9. Nesi, N. et al. The TT8 gene encodes a basic helix-loop-helix domain protein required for expression of DFR and BAN genes in Arabidopsis siliques. Plant Cell 12, 1863-1878 (2000)

10. Nesi, N., Jond, C., Debeaujon, I., Caboche, M. \& Lepiniec, L. The Arabidopsis TT2 gene encodes an R2R3 MYB domain protein that acts as a key determinant for proanthocyanidin accumulation in developing seed. Plant Cell 13, 2099-2114 (2001).

11. Schaart, J. G. et al. Identification and characterization of MYB-bHLH-WD40 regulatory complexes controlling proanthocyanidin biosynthesis in strawbern (Fragaria $\times$ ananassa) fruits. New Phytol. 197, 454-467 (2013).

12. Naval, M. D. M. et al. A WD40-repeat protein from persimmon interacts with the regulators of proanthocyanidin biosynthesis DKMYB2 and DKMYB4. Tree Genet. Genomes 12, 1-11 (2016).

13. Bogs, J., Jaffé, F. W., Takos, A. M., Walker, A. R. \& Robinson, S. P. The grapevine transcription factor vvmybpa1 regulates proanthocyanidin synthesis during fruit development. Plant Physiol. 143, 1347-1361 (2007).

14. Terrier, N. et al. Ectopic expression of VvmybPA2 promotes proanthocyanidin biosynthesis in grapevine and suggests additional targets in the pathway. Plant Physiol. 149, 1028-1041 (2009).

15. Koyama, K. et al. Functional characterization of a new grapevine MYB transcription factor and regulation of proanthocyanidin biosynthesis in grapes. J. Exp. Bot. 65, 4433-4449 (2014).

16. Huang, Y.-F. et al. A negative MYB regulator of proanthocyanidin accumulation, identified through expression quantitative locus mapping in the grape berry. New Phytol. 201, 795-809 (2014).

17. Mellway, R. D., Lan, T. T., Prouse, M. B., Campbell, M. M. \& Constabel, C. P. The wound-, pathogen-, and ultraviolet b-responsive MYB134 gene encodes an R2R3 MYB transcription factor that regulates proanthocyanidin synthesis in poplar. Plant Physiol. 150, 924-941 (2009).

18. Hancock, K. R. \& Rasmussen, S. Expression of the R2R3-MYB transcription factor TaMYB14 from Trifolium arvense activates proanthocyanidin biosynthesis in the legumes Trifolium repens and Medicago sativa. Plant Physiol. 159, 1204-1220 (2012).

19. Gesell, A., Yoshida, K., Tran, L. T. \& Constabel, C. P. Characterization of an apple TT2-type R2R3 MYB transcription factor functionally similar to the poplar proanthocyanidin regulator PtMYB134. Planta 240, 497-511 (2014).

20. Zhou, $H$. et al. Peach MYB7 activates transcription of the proanthocyanidin pathway gene encoding leucoanthocyanidin reductase, but not anthocyanidin reductase. Front. Plant Sci. 6, 908 (2015).

21. Zhu, Q et al. Ectopic expression of the coleus R2R3 MYB type proanthocyanidin regulator gene SsMYB3 alters the flower color in transgenic tobacco. PLOS ONE 10, e0139392 (2015).

22. Tian, J. et al. McMYB12 transcription factors co-regulate proanthocyanidin and anthocyanin biosynthesis in Malus crabapple. Sci. Rep. 7, 43715 (2017).
23. Gopaulchan, D., Umaharan, P. \& Lennon, A. M. A molecular assessment of the genetic model of spathe color inheritance in Anthurium andraeanum (Hort.) Planta 239, 695-705 (2014).

24. Li, C. et al. Characterisation of flavonoids in anthurium spathes and their contribution to spathe colouration. J. Hortic. Sci. Biotech. 88, 208-215 (2013).

25. Collette, V. E., Jameson, P. E., Schwinn, K. E., Umaharan, P. \& Davies, K. M. Temporal and spatial expression of flavonoid biosynthetic genes in flowers of Anthurium andraeanum. Physiol. Plant 122, 297-304 (2004).

26. Gopaulchan, D., Lennon, A. M. \& Umaharan, P. Expression analysis of the anthocyanin genes in pink spathes of anthurium with different color intensities. J. Am. Soc. Hort. Sci. 140, 480-489 (2015).

27. Albert, N. W. et al. Activation of anthocyanin synthesis in Cymbidium orchids: variability between known regulators. Plant Cell Tiss. Organ. Cult. 100, 355-360 (2010).

28. Li, Z., Wang, J., Zhang, X. \& Xu, L. Comparative transcriptome analysis of anthurium "Albama" and its anthocyanin-loss mutant. PLOS ONE 10, e0119027 (2015).

29. Li, C., Qiu, J., Yang, G., Huang, S. \& Yin, J. Isolation and characterization of a R2R3-MYB transcription factor gene related to anthocyanin biosynthesis in the spathes of Anthurium andraeanum (Hort.). Plant Cell Rep. 35, 2151-2165 (2016).

30. Xie, D. Y., Sharma, S. B., Paiva, N. L., Ferreira, D. \& Dixon, R. A. Role of anthocyanidin reductase, encoded by BANYULS in plant flavonoid biosynthesis. Science 299, 396-399 (2003)

31. Han, Y, Vimolmangkang, S, Soria-Guerra, R. E. \& Korban, S. S. Introduction of apple ANR genes into tobacco inhibits expression of both chi and DFR genes in flowers, leading to loss of anthocyanin. J. Exp. Bot. 63, 2437-2447 (2012).

32. Li, X., Li, Z. Y., Cong, H. Q. \& Xu, L. Cloning of ANR gene from Anthurium and relativity analysis of its expression with the spathe color. Mol. Plant Breed. $\mathbf{6}$, 825-830 (2013). in Chinese.

33. Li, C. et al. Flavonoid composition and antioxidant activity of tree peony (Paeonia Section Moutan) yellow flowers. J. Agric. Food Chem. 57, 8496-8503 (2009).

34. Liu, Y. et al. TC-MYBPA an Arabidopsis TT2-like transcription factor and functions in the regulation of proanthocyanidin synthesis in Theobroma cacao. BMC Plant Biol. 15, 160 (2015)

35. Verdier, J. et al. MtPAR MYB transcription factor acts as an on switch for proanthocyanidin biosynthesis in Medicago truncatula. Proc. Natl Acad. Sci. USA 109, 1766-1771 (2012).

36. Gopaulchan, D., Lennon, A. M. \& Umaharan, P. Identification of reference genes for expression studies using quantitative RT-PCR in spathe tissue of Anthurium andraeanum (Hort.). Sci. Hort. 153, 1-7 (2013).

37. Pérez-Díaz, J. R. et al. New member of the R2R3-myb transcription factors family in grapevine suppresses the anthocyanin accumulation in the flowers of transgenic tobacco. Plant Mol. Biol. 90, 63-76 (2016).

38. Matsui, K., Umemura, Y. \& Ohme-Takagi, M. AtMYBL2, a protein with a single MYB domain, acts as a negative regulator of anthocyanin biosynthesis in Arabidopsis. Plant J. 55, 954-967 (2008).

39. Han, Y. et al. Ectopic expression of apple F $3^{\prime} H$ genes contributes to anthocyanin accumulation in the Arabidopsis tt7 mutant grown under nitrogen stress. Plant Physiol. 153, 806-820 (2010).

40. Chen, S., Songkumarn, P., Liu, J. \& Wang, G. L. A versatile zero background Tvector system for gene cloning and functional genomics. Plant Physiol. 150 1111-1121 (2009)

41. Clough, S. J. \& Bent, A. F. Floral dip: a simplified method for Agrobacteriummediated transformation of Arabidopsis thaliana. Plant J. 16, 735-743 (1998).

42. Horsch, R. B., et al. in Plant Molecular Biology Manual (eds Gelvin, S. B. \& Schilperoort, R. A.) A5: 1-9 (Kluwer Academic Publishers, Dordrecht, the Netherlands, 1988).

43. Nakatsuka, T. et al. Identification and characterization of R2R3-MYB and bHLH transcription factors regulating anthocyanin biosynthesis in gentian flowers. Plant Cell Physiol. 49, 1818-1829 (2008)

44. Li, C. et al. Anthocyanin biosynthesis regulation of DhMYB2 and DhbHLH1 in Dendrobium hybrids petals. Plant Physiol. Biochem. 112, 335-345 (2017).

45. Bhargava, A., Mansfield, S. D., Hall, H. C., Douglas, C. J. \& Ellis, B. E. MYB75 functions in regulation of secondary cell wall formation in the Arabidopsis inflorescence Stem. Plant Physiol. 154, 1428-1438 (2010).

46. Jiang, M., Ren, L., Lian, H., Liu, Y. \& Chen, H. Novel insight into the mechanism underlying light-controlled anthocyanin accumulation in eggplant (Solanum melongena L.). Plant Sci. 249, 46 (2016). 
47. Zhang, X. N. \& Mount, S. M. Two alternatively spliced isoforms of the Arabidopsis SR45 protein have distinct roles during normal plant development. Plant Physiol. 150, 1450-1458 (2009).

48. Abrahams, S., Tanner, G. J., Larkin, P. J. \& Ashton, A. R. Identification and biochemical characterization of mutants in the proanthocyanidin pathway in Arabidopsis. Plant Physiol. 130, 561-576 (2002)

49. Feller, A., Machemer, K., Braun, E. L. \& Grotewold, E. Evolutionary and comparative analysis of MYB and bHLH plant transcription factors. Plant J. 66 94e116 (2011)

50. Bai, Y. et al. Flavonoidrelated basic helix-loop-helix regulators, NtAn1a and NtAn1b, of tobacco have originated from two ancestors and are functionally active. Planta 234, 363-375 (2011).

51. Passeri, $V$. et al. The R2R3MYB VVMYBPA1 from grape reprograms the phenylpropanoid pathway in tobacco flowers. Planta 246, 1-15 (2017).
52. Starkevič, P. et al. Expression and anthocyanin biosynthesis-modulating potential of sweet cherry (Prunus avium L.) MYB10 and bHLH genes. PLOS ONE 10, e0126991 (2015).

53. Lai, B. et al. Two LcbHLH transcription factors interacting with LCMYB1 in regulating late structural genes of anthocyanin biosynthesis in Nicotiana and Litchi chinensis during anthocyanin accumulation. Front. Plant Sci. 7, 166 (2016).

54. Liu, X. F. et al. The role of MrbHLH1 and MrMYB1 in regulating anthocyanin biosynthetic genes in tobacco and Chinese bayberry (Myrica rubra) during anthocyanin biosynthesis. Plant Cell Tiss. Organ. Cult. 115, 285-298 (2013).

55. Xie, D. Y., Sharma, S. B., Wright, E., Wang, Z. Y. \& Dixon, R. A. Metabolic engineering of proanthocyanidins through co-expression of anthocyanidin reductase and the PAP1 MYB transcription factor. Plant J. 45, 895-907 (2006).

56. Akagi, T., Ikegami, A. \& Yonemori, K. DkMyb2 wound-induced transcription factor of persimmon (Diospyros kaki Thunb.), contributes to proanthocyanidin regulation. Planta 232, 1045-1059 (2010). 